\title{
A dichotomy in bronchiolitis obliterans syndrome after lung transplantation revealed by azithromycin therapy
}

\author{
B.M. Vanaudenaerde*, I. Meyts* , R. Vos*, N. Geudens", W. De Wever', \\ E.K. Verbeken $^{\S}$, D.E. Van Raemdonck ${ }^{\uparrow, f, * *}$, L.J. Dupont ${ }^{\star, * *, \# \#}$ and G.M. Verleden ${ }^{\star, * *, \# \#}$
}

ABSTRACT: Bronchiolitis obliterans syndrome (BOS) is the most important cause of late mortality following lung transplantation, resulting in major morbidity and a huge burden on healthcare resources. Treatment options are limited, resulting in a mere stabilisation of the lung function decline. Recent introduction of the macrolide antibiotic azithromycin raised new hope after demonstrating lung function improvement in subsets of patients.

The present study aimed to provide an overview of the clinical effects on azithromycin in the setting of BOS after lung transplantation, with special emphasis on the anti-inflammatory actions. Moreover, the authors proposed a new frame of thinking centred on a dichotomy in the pathogenesis and clinical phenotype of BOS. Subsets of BOS patients were identified who do or do not respond to azithromycin (regarding forced expiratory volume in one second (FEV1), bronchoalveolar lavage (BAL) neutrophilia/interleukin-8). These observations have shed new light on the current belief that BOS represents a homogenous clinical entity in which the neutrophil is the main culprit.

Recent clinical observations, supported by research findings, have revealed a dichotomy in the clinical spectrum of BOS with neutrophilic (partially) reversible allograft dysfunction (responding to azithromycin) and fibroproliferative BOS (not responding to azithromycin). This concept is reinforced by unique data obtained in BOS patients, consisting of histology specimens, physical and radiological examination, $F E V_{1}$ and $B A L$ examination.

The acceptance of this dichotomy can improve understanding of the heterogeneous pathological condition that constitutes bronchiolitis obliterans syndrome, thus encouraging a more accurate diagnosis and, ultimately, better tailored treatment for each bronchiolitis obliterans syndrome patient.

KEYWORDS: Azithromycin, bronchiolitis obliterans syndrome, dichotomy, lung transplantation, neutrophils, phenotypes

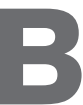

ronchiolitis obliterans syndrome (BOS) is responsible for the vast majority of deaths following lung transplantation (LTx). BOS is diagnosed as an irreversible loss of lung function, after exclusion of other potential causes, and threatens $>50 \%$ of long-term LTx recipients [1]. Symptoms are nonspecific and include an insidious onset of cough and dyspnoea. On chest radiographs, volume loss, subsegmental atelectasis, linear opacities and bronchiectasis are evident. Risk factors for the development of BOS include: acute rejection (AR; including even a single episode of minimal AR); lymphocytic bronchitis/bronchiolitis (LBB); cytomegalovirus (CMV) pneumonitis; human leukocyte antigen mismatching; bacterial/fungal/non-CMV viral infections; decreased immunosuppression; and ischaemia-reperfusion injury [1].

Until 2003, treatment had two main aims. 1) To prevent the development of BOS by administering maintenance immunosuppressive therapy after transplantation. 2) To stop/slow down the decline in forced expiratory volume in one second (FEV1) after the development of BOS by: augmenting or switching immunosuppressive
AFFILIATIONS

Laboratories of *Pneumology, and "Experimental Thoracic Surgery, Katholieke Universiteit Leuven, Depts of "Paediatrics, +Radiology,

${ }^{\text {s} H i s t o l o g y, ~}$

${ }^{f}$ Thoracic Surgery, and

\#\# Respiratory Disease, and

**Lung Transplantation Unit, University Hospital Gasthuisberg, Leuven, Belgium.

\section{CORRESPONDENCE}

G.M. Verleden, Dept of Respiratory Disease and Lung Transplantation Unit, University Hospital Gasthuisberg, Herestraat 49, B-3000 Leuven, Belgium. Fax: 3216346803 E-mail: geert.verleden@ uz.kuleuven.be

Received:

October 122007

Accepted after revision:

March 272008

SUPPORT STATEMENT

G.M. Verleden is supported by the Research Foundation Flanders (Brussels, Belgium), grant numbers: G.0493.04, G.0518.06 and

G.0643.08. B.M. Vanaudenaerde and L.J. Dupont are senior research fellows and R. Vos is a PhD fellow of the Research Foundation Flanders.

STATEMENT OF INTEREST A statement of interest for G.M. Verleden can be found at www.erj.ersjournals.com/misc/ statements.shtml

European Respiratory Journal Print ISSN 0903-1936

Online ISSN 1399-3003 
therapy; better monitoring of the trough levels of the active component of the immunosuppressive drugs; or changing the exposition routes, i.e. inhalation of fluticasone or cyclosporine. Most therapies aim to suppress lymphocyte function and inflammatory responses (adaptive immunity) and have no antifibrotic effect, except, perhaps, for the newer agents such as sirolimus and everolimus. Therefore, therapy is likely to be more effective in the early stages of BOS. Unfortunately, none of these approaches have a significant impact on disease progression and at best all temporarily stabilise FEV1. At the bottom of the list of therapeutic options, re-transplantation emerges as the only strategy that may improve quality of life and lung function in end-stage BOS.

\section{THE FIRST OBSERVATIONAL STUDIES ON AZITHROMYCIN IN PATIENTS WITH BOS}

The recent introduction of the macrolide antibiotic azithromycin raised new hope for BOS patients and their doctors. GERHARDT et al. [2] were the first to use low-dose azithromycin (250 mg three times a week) as an add-on to conventional immunosuppressive therapy in patients with BOS after LTx. That pilot study documented azithromycin treatment in six LTx recipients with established BOS [2]. FEV1 improved in five of the patients with a mean increase of $17.1 \%$ predicted or $0.50 \mathrm{~L}$ over a 4 -month period.

Another study by VERLEDEN et al. [3] included eight LTx patients with established BOS (BOS 3: $n=2 ; \operatorname{BOS} 2$ : $n=2$; BOS 1: $\mathrm{n}=4)$. FEV1 improved in four of the patients with a mean increase of $18.3 \%$ pred or $0.33 \mathrm{~L}$ over a 3 -month period.

A third study by YATES et al. [4] evaluated azithromycin therapy in 20 allograft recipients (BOS 3: $n=10$; BOS 2: $n=2$; BOS 1: $n=6$; BOS 0-p: $n=2$ ). In total, 10 patients responded to the therapy with an overall increase in FEV1 of $14 \%$ pred or $0.11 \mathrm{~L}$ over a 3-month period, which was sustained up to 11 months.

Finally, a study by SHITRIT et al. [5] included 11 LTx patients (BOS 3: $n=1 ; \operatorname{BOS} 2: \mathrm{n}=6$; BOS 1: $n=4$ ). The overall FEV1 had decreased by $1 \%$ after 4 months and $2 \%$ after 10 months. No reversal of BOS could be demonstrated, only an arrest in the progression of the disease.

From these observations it was concluded that the addition of a macrolide antibiotic offers a new and exciting treatment option for BOS. Azithromycin not only arrests further lung function decline, which was the best that could be obtained with other therapies, but even appeared to reverse the decline in lung function in some patients. Out of the 45 patients published in the previously mentioned studies, 19 (42\%) responded to the therapy. The mean FEV1 improvement in these patients was as high as $15 \%$, and this could be at least maintained up to 11 months.

\section{ANTI-INFLAMMATORY EFFECT OF AZITHROMYCIN IN BOS}

The mechanism of action of azithromycin remains obscure, as does the reason why the treatment is only beneficial in some $40 \%$ of patients. Several potential mechanisms can explain the beneficial effect of macrolides, such as an effect on Pseudomonas aeruginosa colonisation and gastro-oesophageal reflux (GER), changes in immunosuppressive trough levels and interaction with the innate immune system (neutrophils/interleukin (IL)-8) were the most prevailing [6].

The beneficial effect of macrolides in different lung disorders and in LTx is believed to be at least partially attributable to the anti-inflammatory properties of the drugs [7]. Recently, a 3-month follow-up study on azithromycin therapy for patients diagnosed with BOS was published [8]. The study went beyond the empiric evaluation of lung function parameters (FEV1/BOS) and was the first to investigate a potential antiinflammatory effect. Emphasis was placed on key players in the innate immune system, especially neutrophils [9] and the potent neutrophil chemoattractant IL-8, measured in the bronchoalveolar lavage (BAL) fluid. In total, 14 LTx patients with BOS (BOS 3: $n=1$; BOS 2: $n=2$; BOS 1: $n=8$; BOS0-p: $n=3$ ) were included. In the study population as a whole, azithromycin therapy resulted in a significant mean increase in FEV1 by $0.31 \mathrm{~L}$ (or $13 \%$ ) after 3 months of therapy. Of equal importance was the fact that this improvement was accompanied by a significant decrease of both BAL neutrophils and BAL IL-8 (fig. 1a-c). The possible involvement of GER, bacterial growth (Mycoplasma pneumoniae, Chlamydia pneumoniae or $P$. aeruginosa) and varying immunosuppressive trough levels as inducers of the neutrophilic inflammation, and, consequently, the target of azithromycin, could not be confirmed, although it is accepted that a vast number of identified and unidentified conditions may contribute to the neutrophilic inflammation observed in BOS [11, 12].

First, a link between Pseudomonas colonisation, airway neutrophilia and BOS has been hypothesised [12, 13], and it was assumed that Pseudomonas colonisation is a prerequisite for azithromycin responsiveness. GERHARDT et al. [2] demonstrated that four out of six patients had Pseudomonas colonisation in the airways or sinuses. However, VERLEDEN and DuPONT [3] reported no difference in the effect of azithromycin on FEV1 between colonised and noncolonised patients. Furthermore, SHITRIT et al. [5] found positive airway Pseudomonas cultures in nine out of 11 patients without one single patient responding to azithromycin. It is also possible that airway colonisation is not always detected, since quorumsensing signal proteins have been detected in so-called stable, noncolonised LTx patients [14, 15]. Thus, Pseudomonas colonisation may indeed contribute to neutrophilic inflammation [12] and possibly BOS, yet not fully explain the response to azithromycin. For this reason it is probably not the discriminating factor between responders and nonresponders.

Secondly, a positive effect on GER may also explain the effect of azithromycin, as it is a known motilin agonist [16]. GER is linked with acid aspiration of gastric contents, inducing lung injury, sustained airway inflammation and hyperresponsiveness to the lung, which may cause reversible allograft dysfunction after LTx [17-19]. Moreover, LTx increases the incidence of GER, as measured by 24-h pH monitoring, although most transplant patients with GER are asymptomatic [20]. However, in the studies of VERLEDEN and co-workers [21, 22], all patients were treated with tacrolimus, which is known to improve gastric discomfort after LTx, arguing against GER. A recent observation showed that BAL levels of pepsin, a marker of gastric aspiration, were significantly lower in patients treated with azithromycin, when compared with 

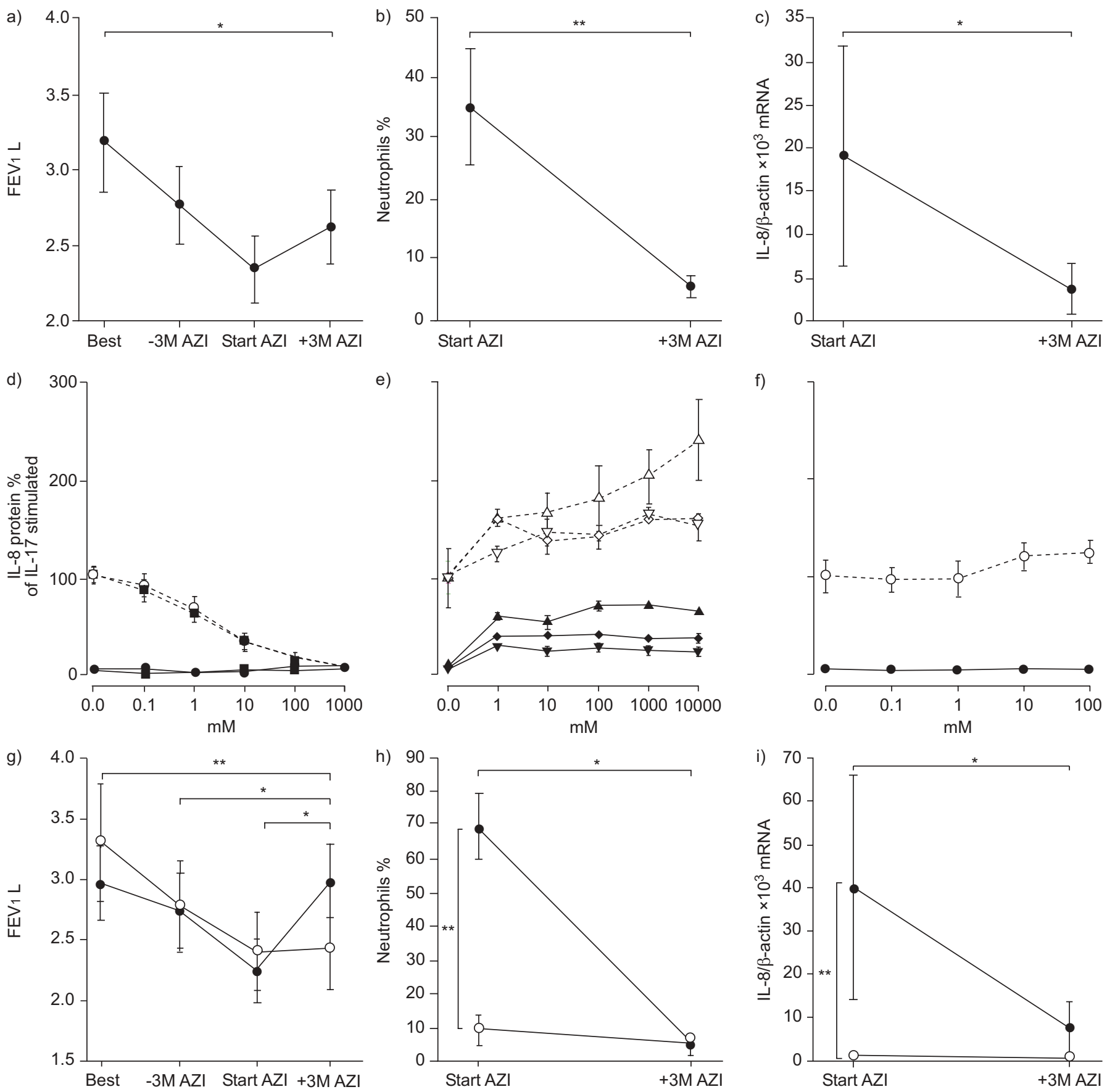

FIGURE 1. a-c) The anti-inflammatory effect of azithromycin (AZI) in all patients with established bronchiolitis obliterans syndrome (BOS). a) Evolution of forced expiratory volume in one second (FEV1) at the best (Best) post-operative value obtained, 3 months before AZI initiation (-3M AZI), the start of AZI (Start) and after 3 months of treatment (+3M AZI). b) Neutrophils and c) interleukin (IL)-8 mRNA (ratio of IL-8 over $\beta$-actin mRNA) levels in bronchoalveolar lavage (BAL) at the start of AZI and after 3 months of treatment. Total study population $n=14$. Data are presented as mean \pm SEM. ${ }^{*}: p<0.05 ;{ }^{*}: p<0.01$. $\left.d-f\right)$ The anti-inflammatory effect of AZI in vitro on human airway smooth muscle cells (HASMC). The effect of d) macrolides, e) immunosuppressive agents and f) dexamethasone (DEX) on the IL-8 production in nonstimulated (—) and IL-17-stimulated (Stim.;- - - - -) HASMC. IL-8 is expressed as mean \pm SEM and the IL-17-stimulated group was set referentially as $100 \%$ of IL-8 protein. HASMC were either stimulated (or not) with IL-17 (10 ng. $\left.\mathrm{mL}^{-1}\right)$ for $24 \mathrm{~h}$. Modulators were added $30 \mathrm{~min}$ in advance. d) $\mathrm{O}$ : IL-17 plus AZI; $\square$ : IL-17 plus erythromycin; • : AZI; $\mathbf{\square}$ : erythromycin.

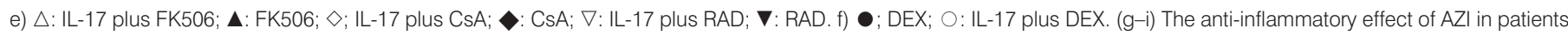
with established BOS according to therapy response. g) Evolution of FEV1 at the best (BEST) post-operative value obtained, 3 months before AZI initiation (-3M AZI), the start of AZI (Start) and after 3 months of treatment (+3M AZI). h) Neutrophils and i) IL-8 mRNA (ratio of IL-8 over $\beta$-actin mRNA) levels in BAL at the start of AZI and after 3 months of treatment. Responders $(\bullet ; n=6)$ and nonresponders $(O ; n=8)$ to the therapy are based on an FEV1 improvement of $10 \%$. Data are presented as mean \pm SEM. ${ }^{*}: p<0.05$; **: $p<0.01$. a-c and g-i) Reproduced and modified from [8] with permission from the publisher. $d-f$ ) Reproduced and modified from [10] with permission from the publisher 
patients who were not [23]. As a consequence, GER can be implicated in the action of azithromycin but is probably not the only explanation as to the beneficial effect and it certainly does not discriminate between responders and nonresponders.

The effect of macrolide therapy, specifically involving neutrophils, has been further studied in vitro in human airway smooth muscle cells [10] and in bronchial epithelial cells [24], adding further evidence to the plausibility that azithromycin acts via an anti-inflammatory mechanism in BOS. In a recent study, VANAUDENAERDE et al. [10] demonstrate that azithromycin inhibits the IL-17-induced IL-8 production in these cells (fig. 1d-f), and may inhibit neutrophil activation and recruitment. Immunosuppressive agents classically used in the setting of LTx even seem to further enhance the IL-17-induced IL-8 production, whereas steroids had no significant suppressive effect at all (fig. $1 \mathrm{~d}-\mathrm{f}$ ). These findings give rise to speculation about potential immunosuppressive over-treatment in patients with BOS, which is a controversial issue. In the aforementioned study, the principal difference between immunosuppressive drugs/steroids and macrolides was the inhibition of the different mitogen-activated protein kinase (MAPK), p38-MAPK, extracellular regulated kinase and Janus-N-terminal kinase, and the reduction of oxidative stress (as measured by 8 -isoprostane) by azithromycin but not by steroids or any of the tested immunosuppressive drugs [10]. Indirectly, these findings lead to the conclusion that, in a simplified view, macrolides appear to specifically reduce inflammation by inhibiting components of the innate immune system activation, whereas immunosuppressive agents suppress lymphocytes, key players in the adaptive immune system, without any effect on the ongoing neutrophilic inflammation. By contrast, conventional immunosuppressive agents even seem to enhance chemokine production, and potentially neutrophil attraction.

\section{A DICHOTOMY IN BOS REVEALED BY AZITHROMYCIN}

It is clear that not all LTx patients with established BOS benefit from this macrolide therapy as the documented response rate, which is arbitrarily defined as an FEV1 increase of $\geqslant 10 \%$, until now was 25 out of 59 published patients, which is comparable to observations in other respiratory diseases such as cystic fibrosis [25]. The discriminating variables in the dichotomy of responders and nonresponders were the BAL neutrophilia, BAL IL- 8 and the post-operative day at which the therapy was started [8]. Responders developed BOS early ( \pm 8 months) following transplantation, while nonresponders developed BOS much later $( \pm 42$ months). The responders (median FEV1 improvement of $0.60 \mathrm{~L}$ ) were characterised by a significantly higher level of neutrophils ( \pm 8 -fold increase) and IL- 8 ( \pm 10 -fold increase $)$ in BAL obtained prior to commencing azithromycin therapy. Azithromycin treatment dramatically reduced the neutrophil and IL-8 levels to baseline values, as obtained in the nonresponder group of patients (fig. $1 \mathrm{~g}-\mathrm{i}$ ). In fact, the presence of increased airway neutrophilia $(>15 \%)$, in the absence of infection, may predict whether azithromycin therapy will have a positive effect on FEV1. This assumption is further supported by a positive correlation between initial BAL neutrophilia/BAL IL-8 and the changes in FEV1 observed during the study period $(r=0.79 / r=0.76)$. In a simplified frame of thinking, whether a patient with BOS will improve with azithromycin therapy can almost be predicted just by evaluating the BAL neutrophilia and IL-8.

This new concept of dichotomy in BOS with the existence of at least two phenotypes (neutrophilic reversible allograft dysfunction (NRAD) and fibroproliferative BOS (fBOS)) is documented below in seven patients diagnosed with BOS (figs 2-4).

Patient 1 (fig. 2a) underwent LTx in July 1992 and represents an example of the NRAD phenotype in the era before azithromycin was introduced. While in BOS 1, open lung biopsy (OLB) 2 yrs after LTx (fig. 2d) demonstrated dense inflammatory infiltrates linked with fibroproliferation restricted to the membranous bronchioles (MB). The patient was intermittently treated with augmented doses of oral and intravenous steroids, but died of slowly progressive BOS in November 2000. The autopsy (AUT; fig. 2e) revealed complete fibrous obliteration of the MBs, almost no residual inflammation, and post-obstructive fibrosis and atelectasis. The patient represented the spontaneous evolution from inflammatory active obliterative bronchiolitis (OB) lesions, to fibrotic active $\mathrm{OB}$ lesions through to pure inactive fibroproliferative $\mathrm{OB}$.

In August 1991, patient 2 (fig. 2b) underwent LTx and was retrospectively diagnosed with BOS0-p in September 1996. The patient was initiated on azithromycin therapy in January 2001, 2 yrs after the diagnosis of BOS 2 (December 1998). There was a clear increase in FEV1 over time, and the patient's condition improved to BOS 1, but FEV1 did not normalise. The present authors speculate that the patient started with pure inflammatory active $\mathrm{OB}$ lesions, which evolved to fibrotic active $\mathrm{OB}$ lesions and to inactive OB. The improvement in terms of FEV1 pointed to the inflammatory active component (NRAD), which was successfully treated with azithromycin (reflected by increasing FEV1). The absence of normalisation of FEV1 pointed to the fibrotic active components of $\mathrm{OB}$, indicating co-existence of inflammatory active and fibrotic active $\mathrm{OB}$ lesions. This patient suffered from mild airway fibrosis, as demonstrated on transbronchial biopies (TBB), which hampered the complete recovery of the FEV1.

Patient 3 (fig. 2c) underwent LTx in October 2004. This patient had participated in a previous clinical trial [8] and received azithromycin therapy directly after the onset of BOS. AR (grade A1) was diagnosed in January 2005, with 2\% lymphocytes and $12 \%$ neutrophils present in BAL. By the end of March 2005, an LBB was found on TBB and BAL revealed $2 \%$ lymphocytes and $97 \%$ neutrophils, without signs of infection. The patient did not recover despite high doses of corticosteroid therapy and was BOS 1 in April 2005. At that time, azithromycin therapy was started and the patient fully recovered in terms of lung function (NRAD). BAL neutrophils were quantified at three later time-points for the purpose of routine follow-up and decreased to 7, 2 and 0\% in May 2005, September 2005 and March 2006, respectively.

Patient 4 (fig. 3a) represents an example of the fBOS phenotype. The patient underwent LTx in May 1998 and performed well for $>4$ yrs. In November 2002 an AR (grade A2) was diagnosed. This was treated with a 3-day course of high-dose i.v. steroids, resulting in a partial restoration of the FEV1, which was immediately followed by a rapid and progressive 

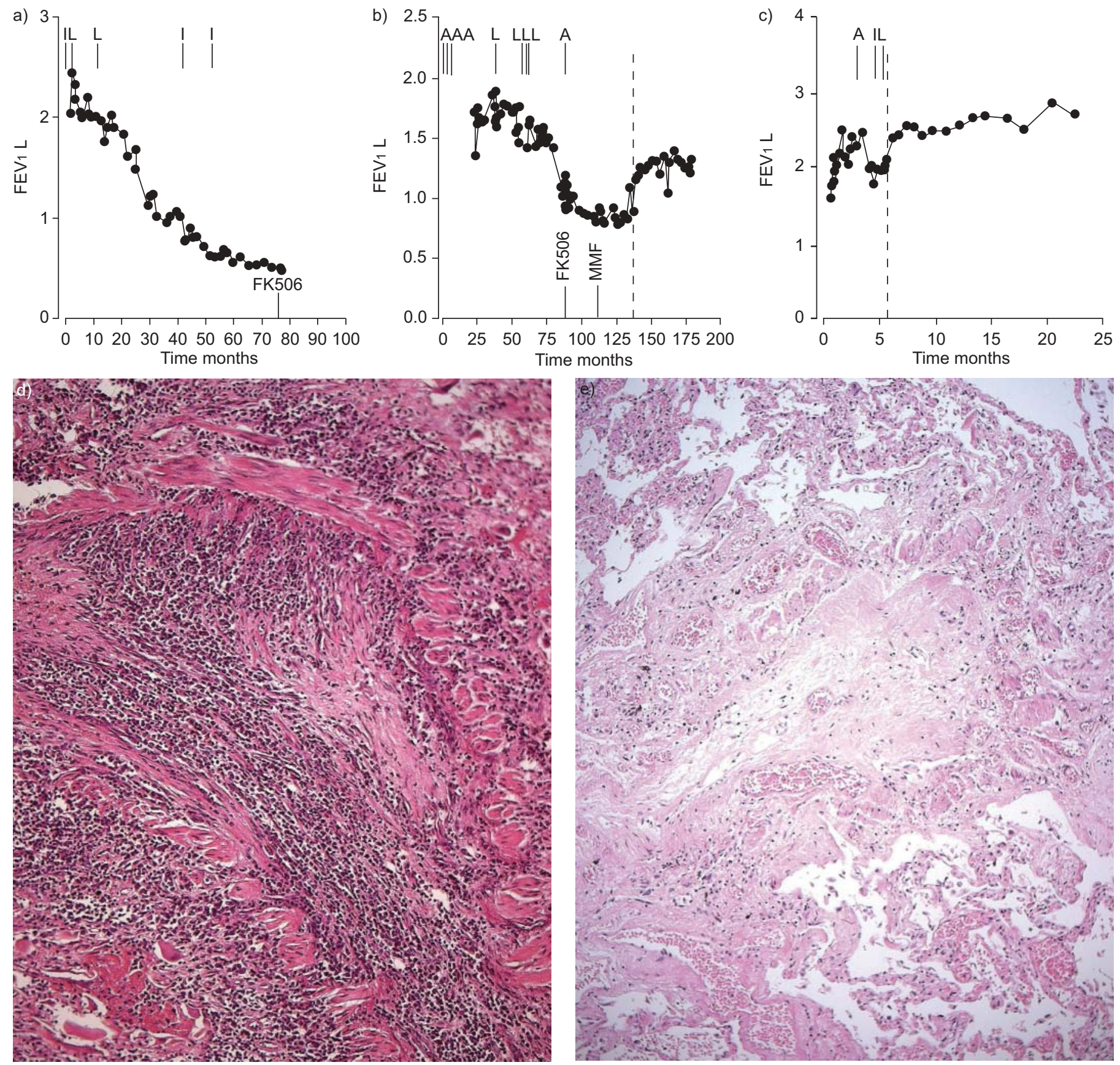

FIGURE 2. Lung function and histological evidence of the dichotomy in bronchiolitis obliterans syndrome with phenotype neutrophilic reversible allograft dysfunction. a) Patient 1, b) patient 2 and c) patient 3. - - - -: start of azithromycin therapy. A: acute rejection; I: infection; L: Iymphocytic bronchitis/bronchiolitis; FK506: tacrolimus; MMF: mycophenolate mofetil; FEV1: forced expiratory volume in one second. d) Histology sample obtained from patient 1 at open lung biopsy after 30 months. e) Histology sample obtained from patient 1 at autopsy biopsy after 100 months.

fall of the FEV1. BAL demonstrated no neutrophilic inflammation (6\% lymphocytes, 3\% neutrophils). Repeated high doses of steroids and a therapeutic switch from azithioprine and cyclosporine to mycophenolate mofetil and tacrolimus proved ineffective. In January 2003, BOS 1 was diagnosed and azithromycin therapy was initiated; the patient failed to respond. A course of rATG and sirolimus started in February and March 2003, respectively, proved ineffective. BAL neutrophilia remained low on several routine investigations (10, 5 and 5\% in May 2003, April 2005 and June 2007, respectively).
In September 1998, patient 5 (fig. 3b) underwent LTx. In a 5month period prior to BOS onset no infection or rejection was diagnosed. At the time BOS developed in August 2004, GER was excluded by $24-\mathrm{h} \mathrm{pH}$ monitoring. The BAL neutrophilia was low (9\%) and there was no evidence of Chlamydia, Mycoplasma or active CMV disease. TBB showed neither AR nor LBB, and lung auscultation was completely normal. Azithromycin treatment was initiated in September 2004 but the patient did not respond. It was speculated that fibroproliferative $\mathrm{OB}$ was responsible for the 


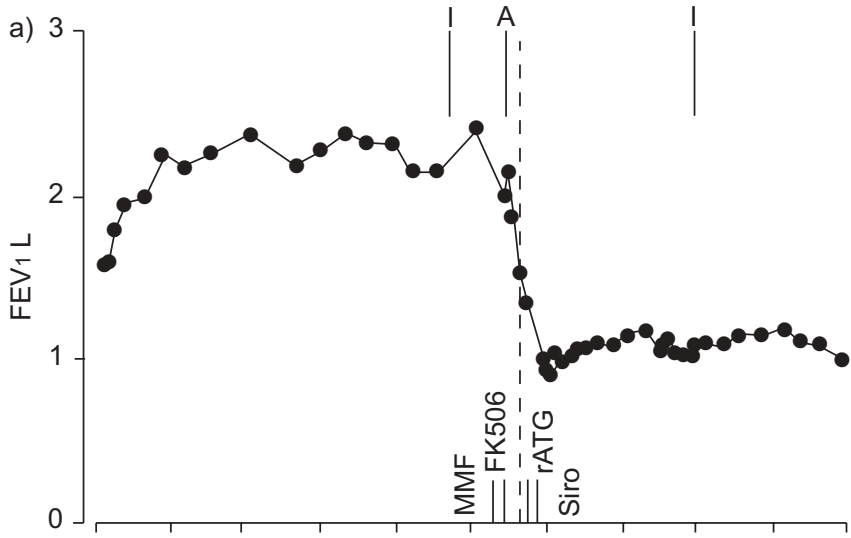

b) 4 -
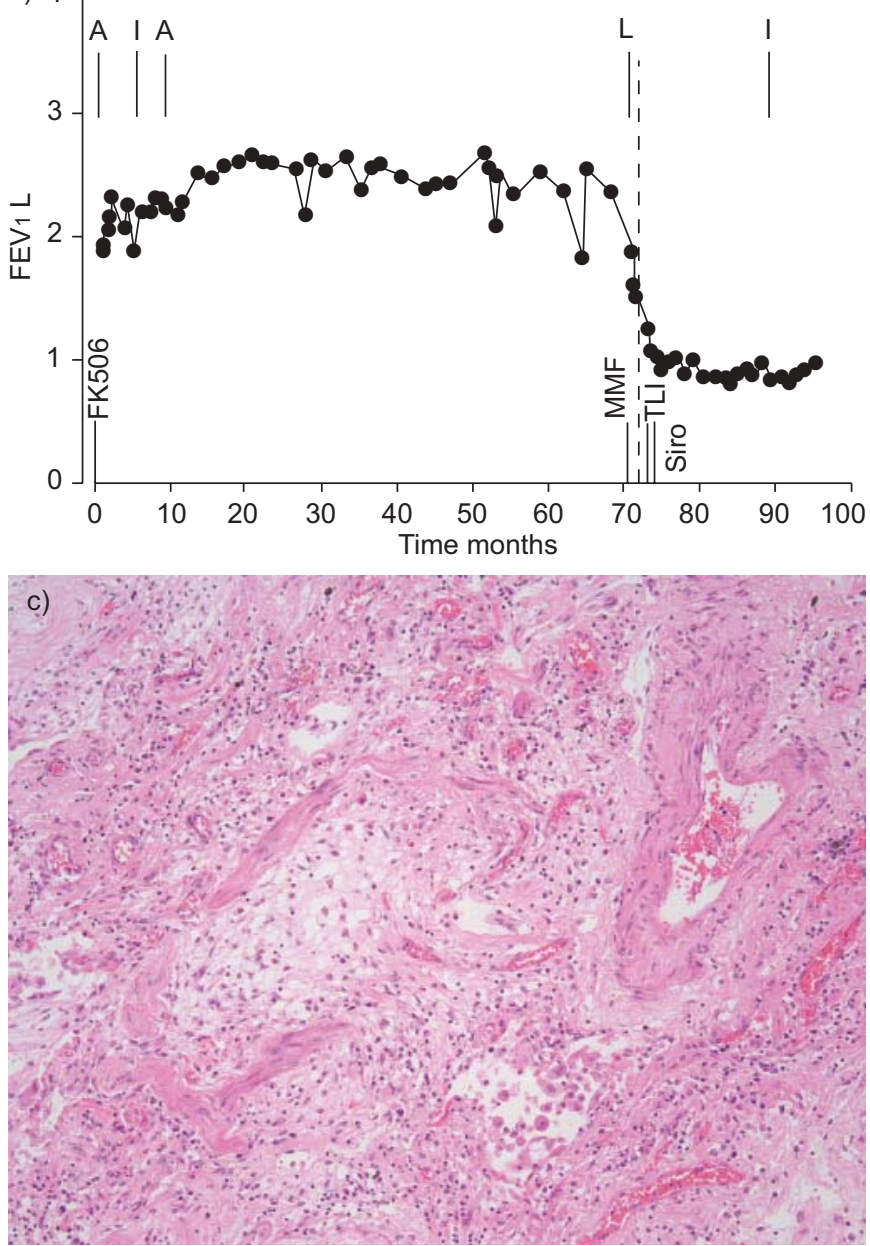

FIGURE 3. Lung function and histological evidence of the dichotomy in bronchiolitis obliterans syndrome (BOS) with phenotype fibroproliferative BOS (fBOS). a) Patient 4 and b) patient 5. - - - -: start of azithromycin therapy. A: acute rejection; L: lymphocytic bronchitis/bronchiolitis; FK506: tacrolimus; MMF: mycophenolate mofetil; rATG: rabbit anti-thymocyte globuline; Siro: sirolimus; TLI: total lymphoid irradiation. c) Histology sample obtained from patient 5 at autopsy biopsy after 100 months

rapid deterioration in FEV1. Unfortunately, the patient died in December 2006 due to BOS. An autopsy section revealed inactive $\mathrm{OB}$ lesions (fig. 3c).
In December 2001, patient 6 (fig. 4a) underwent LTx. In June 2002, the FEV1 started to decline and reached BOS 0-p. BAL neutrophilia was high $(86 \%)$ and BAL culture demonstrated moderate growth of $P$. aeruginosa, which was considered as a colonisation. In November 2002, azithromycin treatment was started and the patient completely recovered from BOS by January 2003 showing all the characteristics of the NRAD phenotype. Following this, neutrophilia remained low at 2.5 and 4\% in December 2003 and 2004, respectively. By May 2005, the patient had started to develop the rapid fBOS phenotype, with a low BAL neutrophilia (5\%). In May 2005, the patient was at BOS 0-p but by August 2005 it was BOS 3. In August 2005, an AR grade AxB3 was diagnosed and treated with high doses of i.v. steroids. BAL neutrophilia was low (5\%) but BAL culture still showed moderate presence of $P$. aeruginosa. The patient underwent re-transplantation in May 2006 and histology of the explanted lung demonstrated inactive OB lesions.

Patient 7 (fig. 4b) also consecutively developed the NRAD and fBOS phenotypes. The patient underwent LTx in July 1997 and experienced several ARs and an episode of LBB early after transplantation. The patient was free of BOS for nearly $5 \mathrm{yrs}$ but in November 2001 the patient developed BOS 0-p and finally BOS 2 in September 2002. Azithromycin therapy was initiated in January 2003. Within a few weeks FEV1 improved to BOS 0 . The patient remained stable for $>18$ months. In August 2004, while still being treated with azithromycin, the patient again developed BOS (BOS 0-p in August 2004, BOS 1 in February 2005 and BOS 2 in October 2005), with the absence of neutrophils in BAL and no FEV1 response to changes in immunosuppressive drug regimen (higher doses of oral steroids, addition of sirolimus).

The findings of a recent clinical study [8] and the seven cases in the present study illustrate this BOS dichotomy in more detail, which is summarised in figure 5 and table 1 . The first phenotype, NRAD, commonly develops rather early after transplantation and can be clinically discriminated by the presence of crackles on lung auscultation and by increased sputum production, and further diagnosed by neutrophilic inflammation in the BAL $(>15 \%)$, without signs of AR nor infection, leading to a protracted disease course with slowly declining FEV1. When treated with azithromycin early in the disease course, these patients are likely to recover from declined pulmonary function. This recovery casts a shadow on the classical definition of BOS, which implicates an irreversible loss of lung function. The second phenotype, fBOS, is most likely to develop much later after transplantation. Although fBOS may progress rapidly, little or no inflammation (at least neutrophilic) in the BAL is present. Clinically, crackles and, at least initially, excessive sputum production are absent. This is an irreversible type of declining FEV1 for which, unfortunately, no therapy (including azithromycin) is currently effective; thus, the only option is to try and slow down the decrease in FEV1 (by classical changes in immunosuppressive treatment) and advise for re-transplantation in due course.

\section{HISTOLOGICAL PRESENTATION OF THE DICHOTOMY}

How will the pathologist interpret this dichotomy of BOS, especially since a spectrum of $\mathrm{OB}$ lesions has already been formulated, ranging from active (mononuclear infiltrate) to inactive (fibrotic lesions) [1, 26]. The first critical remark has to 

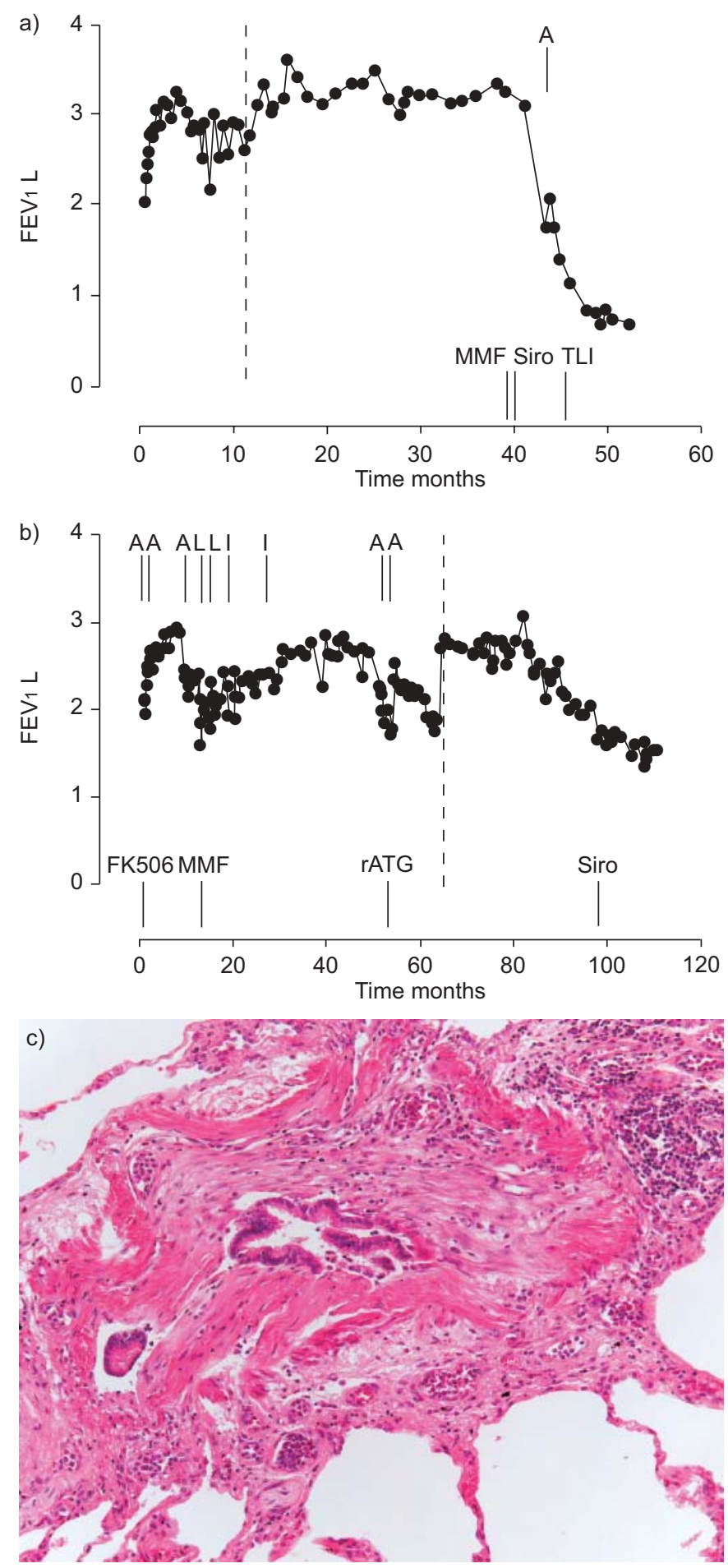

FIGURE 4. Lung function and histological evidence of the dichotomy in bronchiolitis obliterans syndrome (BOS) with phenotype fibroproliferative BOS (fBOS) and neutrophilic reversible allograft dysfunction (NRAD). a) Patient 6 and c) patient 7. - - - start of azithromycin therapy. A: acute rejection; L: lymphocytic bronchitis/bronchiolitis; I: infection; FK506; tacrolimus; MMF: mycophenolate mofetil; rATG: rabbit anti-thymocyte globuline; Siro: sirolimus; TLI: total lymphoid irradiation. c) Histology sample obtained from patient 6 at autopsy biopsy after 100 months. be made about the term "inactive lesions", as it is ambiguous to allude to an inactive process (OB) in the presence of ongoing active fibrosis which is most harmful. Bearing the dichotomy in mind, it might be preferable to use three terms to describe pathological findings in BOS: 1) inflammatory active OB; 2) fibrotic active $\mathrm{OB}$; and 3) inactive $\mathrm{OB}$. Inflammatory active lesions are characterised by a prominent peribronchiolar infiltrate of mononuclear cells (macrophages and lymphocytes). Fibrotic active lesions indicate active infiltration of fibroblasts, which may persist in the absence of ongoing alloimmune stimuli. Invading fibroblasts and myofibroblasts may migrate into the luminal exudates and form intraluminal fibromycoid granulation tissue polyps [1]. Finally, the inactive lesions are characterised by collagenous connective tissue, hence scarring of the bronchiolar wall occurs in the absence of significant inflammation or active fibrosis. Eventually, inactive $\mathrm{OB}$ is the complete fibrotic obliteration of the airway lumen without residual epithelium, with at least partial disruption of the elastic lamina and smooth muscle layer.

A continuum from inflammatory active over fibrotic active to inactive lesions can be present at one given time-point within a single patient. All three types of lesions may occur as a pure or as an extended form, and, in some patients, may only affect the bronchial tree regionally. An important observation is that no asynchronous lesions are found, meaning that if inflammatory active, fibrotic active or inactive $\mathrm{OB}$, or a form somewhere inbetween, are present at a certain time-point, this is the same for all lesions throughout the lung. When transected, the lesions may be excentric or concentric or may completely obliterate the lumen [27].

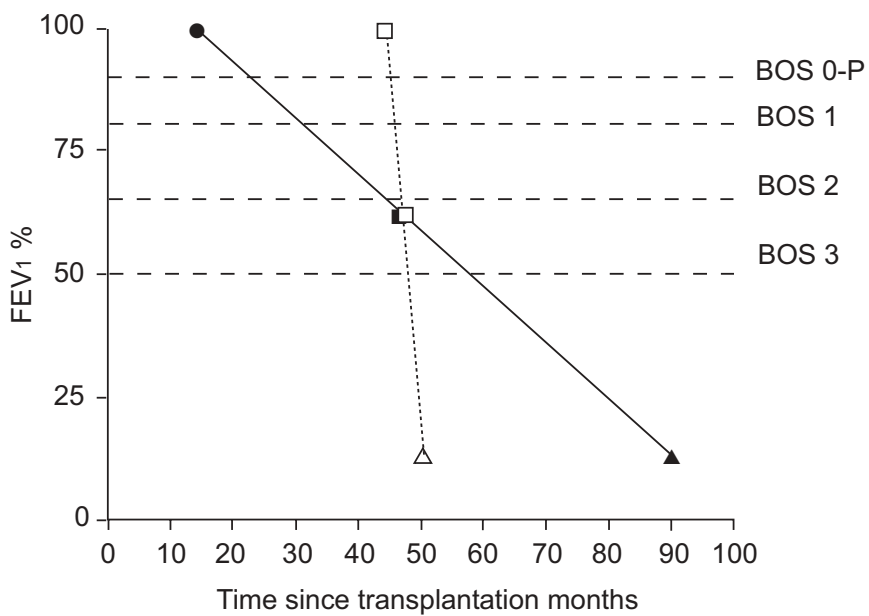

FIGURE 5. Characterisation of the dichotomy in bronchiolitis obliterans syndrome (BOS). Neutrophilic reversible allograft dysfunction (NRAD; $\longrightarrow$ ) develops slowly over several years \pm 8 months following transplantation. Fibroproliferative BOS (fBOS; ...........) develops rapidly, within 6 months, and \pm 42 months following transplantation. FEV1: forced expiratory volume in one second. - neutrophils and interleukin (IL)-8 present in bronchoalveolar lavage (BAL) and inflammatory active obliterative bronchiolitis (OB) lesions; $\mathbf{a}$ : neutrophils and IL-8 in BAL and inflammatory/fibrotic active OB; $\mathbf{\Lambda}$; no neutrophils or IL-8 in BAL and in active OB; $\square$ : no neutrophils or IL-8 in BAL and inflammatory, fibrotic active OB; $\triangle$ : no neutrophils or IL-8 in BAL and inactive OB. 
Figure 5 shows both histology and clinical presentation. The NRAD phenotype correlates histologically to inflammatory active lesions in the early stages (e.g. OLB of patient 1 ). If left untreated, this will gradually and slowly evolve into fibrotic active and eventually inactive lesions (e.g. AUT of patient 1). The histological appearance of OLB in the early stage of fBOS is still unknown but rapidly progresses to inactive OB lesions (AUT and explant lung biopsy of patients 5 and 6). Both phenotypes display comparable lesions in end-stage disease, i.e. fBOS in patient 6 and NRAD in patient 1 .

A second critical comment should be raised with regard to discrepancies in the predominant inflammatory cell type observed in the BAL versus the bronchial biopsies. There appears to be an association between the presence of mononuclear cell infiltrate (macrophages and lymphocytes) on histological examination of the airways and predominantly neutrophilic inflammation in the BAL (fig. 6). The paradox between a chronic mononuclear cell tissue infiltrate in association with a polymorphonuclear exudate remains unexplained. However, the association is also known in other conditions, such as an active stage of rheumatoid arthritis. In this particular condition, a synovial biopsy characteristically shows a chronic inflamed synovium infiltrated by lymphocytes and plasma cells, with some degree of follicular hyperplasia. At the same time, an aspiration of the joint presents an inflammatory exudate with mainly polymorphonuclear leukocytes, which may even suggest the possibility of septic arthritis [28]. The difference between tissue infiltration with lymphocytes/plasmocytes (bronchial wall, synovium) and aspiration of cells (neutrophils) in empty spaces (bronchial lumen, synovial space) remains enigmatic.

\section{RADIOLOGY}

According to the criteria described by DE JONG et al. [29], further radiological evaluation of NRAD and fBOS assessing air trapping, mosaic perfusion, consolidation, mucus plugging, airway wall thickening and bronchiectasis was performed in patients who took part in the previous clinical study [8] (within 1 week of inclusion; W. de Wever, personal communication). NRAD (in contrast to fBOS) was found to be associated with bronchiectasis (40 versus $0 \%$ ), airway wall thickening (60 versus $14 \%$ ) and mucus plugging (43 versus $20 \%$ ), whereas fBOS (in contrast to NRAD) was characterised by air trapping (71 versus $40 \%$ ) and consolidation (43 versus 20\%). Mosaic pattern was present in $\sim 40 \%$ of the cases, equally distributed in both phenotypes. Thus, bronchiectasis, mucus plugging and airway wall thickening are more common in NRAD, whereas air trapping and consolidation are more prominent in fBOS, although the distinction is not clear cut (table 1).

\section{PHARMACOLOGY OF AZITHROMYCIN}

Macrolides are a group of antibiotics derived from the Streptomyces spp., which consist of a common lacton ring with one or more sugars attached. Independent of their antimicrobial activity, macrolides possess anti-inflammatory properties that may contribute to the clinical benefits observed in patients with chronic airway inflammation [7]. The use of low-dose macrolide therapy was first described in diffuse panbronchiolitis [7], followed by cystic fibrosis [7, 30], asthma [31] and chronic obstructive pulmonary disease [32] due to its antiinflammatory potency.

So far, in LTx, azithromycin has only been used in a dose of $250 \mathrm{mg}$ every other day, based on the experience of azithromycin in other lung disorders. Current experience has demonstrated that if $250 \mathrm{mg}$ is not effective, a higher dose of $500 \mathrm{mg}$ will not be effective either. Furthermore, it has been demonstrated in cystic fibrosis patients that a weekly dose of $1,000 \mathrm{mg}$ has the same effect on FEV1 but more side-effects were observed [33].

The explanation for using only azithromycin in LTx can be found in the inhibition of P-glycoprotein (P-gp) and the hepatic or intestinal mixed-function oxidase system (CYP3A4, an isoform of cytochrome P-450), which are involved in absorption, distribution and elimination in the gastrointestinal epithelium of immunosuppressive agents in LTx patients [34]. Erythromycin and clarithromycin strongly inhibit P-gp and CYP3A4, thereby increasing the trough levels of tacrolimus/cyclosporine [35], resulting in more pronounced sideeffects. Azithromycin only minimally interferes with CYP3A4 and P-gp and consequently does not affect trough levels of tacrolimus/cyclosporine [15]. Only one recent case has reported an interaction on tracrolimus blood levels [36].

Another important feature is the intracellular accumulation, especially by azithromycin, which extends to 226 times the extracellular concentration, leading to a concentration gradient (internal/external) 26 times higher than erythromycin in macrophages [37]. This may also explain the improved delivery to the site of inflammation and higher potency compared with erythromycin [37].

In previous studies, and in the present authors' experience, prolonged use of azithromycin was well tolerated and the adverse effects (nausea, diarrhoea and vomiting) were generally mild to moderate and caused discontinuation of the drug in $<10 \%$ of the patients.

Resistance to macrolide antibiotics may develop in numerous pathogens, including Streptococci spp., Streptococcus pneumoniae, Staphylococcus aureus and nontuberculous mycobacteria. However, to date, this has not been reported.

To prevent resistance, stopping azithromycin therapy could be a consideration, although this has not yet been documented in responders. In the present authors' experience, NRAD patients who show improvement with azithromycin therapy do not want to stop it. However, in patients not responding to azithromycin, the therapy is usually stopped after 3 months.

In some patients, however, an arrest in the decline of FEV1 was documented, which remained stable after stopping the treatment. This may allude to a potential anti-proliferative effect of azithromycin for the fBOS phenotype, but this has not been profoundly investigated yet.

\section{DISCUSSION}

The introduction of azithromycin in LTx is a turning point in the treatment of BOS as it has been shown to improve lung function in a large subset of patients, and by doing so it has also revealed the existence of a dichotomy in BOS. The acceptance of this dichotomy offers a feasible frame of thinking 


\begin{tabular}{|c|c|c|}
\hline \multirow[t]{2}{*}{ TABLE 1} & \multicolumn{2}{|c|}{$\begin{array}{l}\text { eristics of neutrophilic reversible allograft dysfunction (NRAD) and fibroproliferative } \\
\text { drome ( } \mathrm{fBOS} \text { ) }\end{array}$} \\
\hline & NRAD & fBOS \\
\hline Inflammation (BAL) & Neutrophilic airway inflammation & No airway inflammation \\
\hline Clinically & Coarse crackles and increased sputum production & No coarse crackles, minimal sputum \\
\hline Time of onset & Early onset after transplantation & Late onset after transplantation \\
\hline Histology & Initially inflammatory, ends in pure fibrosis & Pure fibrosis \\
\hline Radiology & $\begin{array}{c}\text { Bronchiectasis, airway wall thickening and } \\
\text { mucus plugging }\end{array}$ & $\begin{array}{l}\text { Air trapping and } \\
\text { consolidation }\end{array}$ \\
\hline Azithromycin & Reversible (effective) & Irreversible (ineffective) \\
\hline
\end{tabular}

BAL: bronchoalveolar lavage.
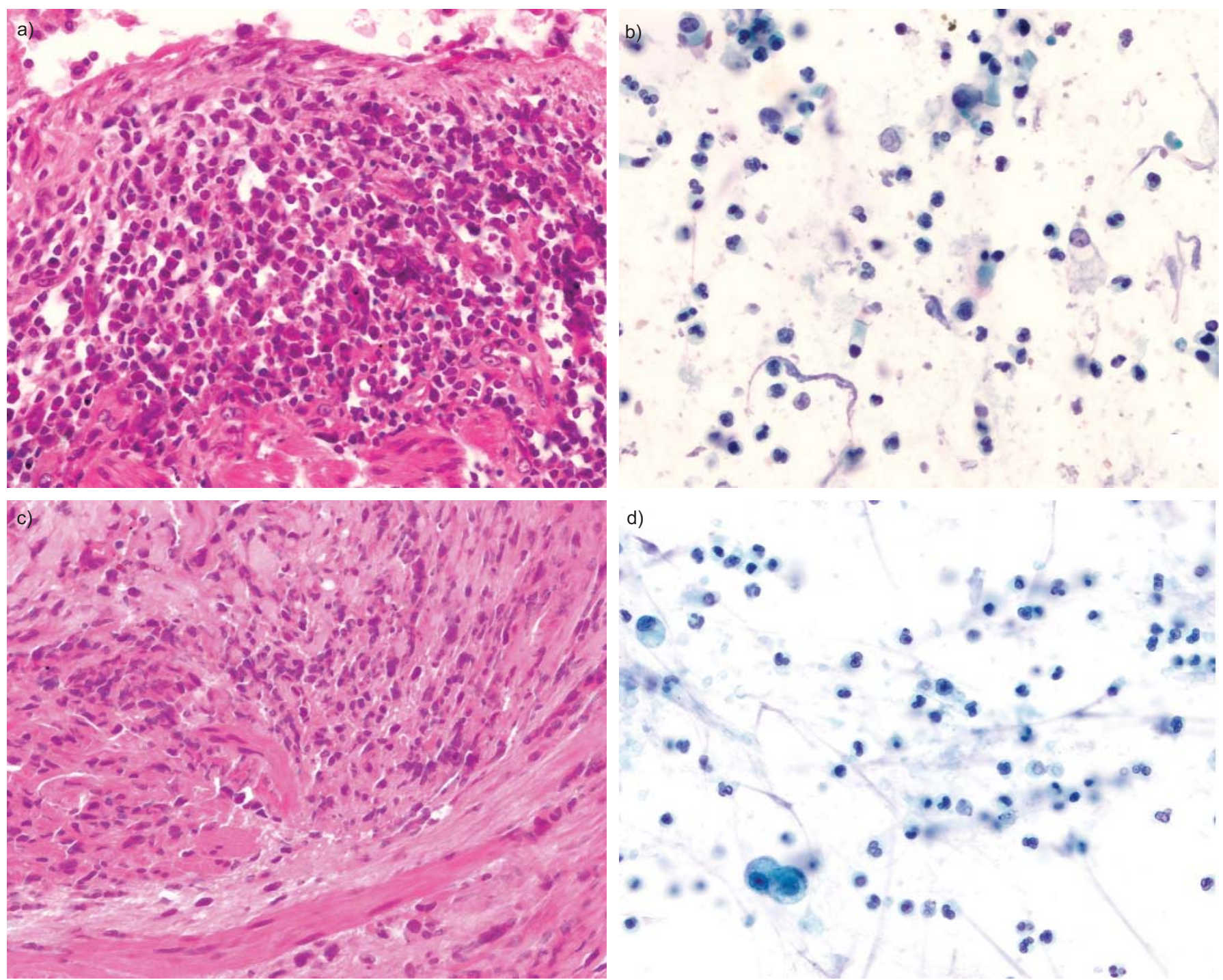

FIGURE 6. Different types of inflammation for the neutrophilic reversible allograft dysfunction (NRAD) phenotype depending on the compartment in the lung. a) The early stage of NRAD in patient 1 demonstrated predominantly lymphocytes and macrophages on the open lung biopsy obtained on October 24, 1994. b) Neutrophils in the bronchoalveolar lavage (BAL) obtained 1 month later. Patient 2 confirmed these finding of increased c) lymphocytes/macrophages on transbronchial biopies and d) neutrophils in BAL, both performed on November 29, 1994 
for understanding the unique disease course in the individual patient diagnosed with BOS. The two phenotypes described in the present study are NRAD and fBOS (table 1). Clinically, this implies that if a patient starts developing a decline in FEV1, which to date has indicated BOS, and shows signs of increased BAL neutrophils (>15\%), BAL IL- 8 mNRA, inflammatory active lesions on histology, increased sputum production, coarse crackles, presence of airway wall thickening, mucus plugging and bronchiectasis on a computed tomography (CT) scan, the patient is likely to have a good prognosis when started on azithromycin. In contrast, if there is no evidence of increased BAL neutrophilia or IL-8 mRNA, minimal sputum production and little or no coarse crackles in the presence of air trapping and consolidation on CT, the patient's condition is likely to deteriorate. Re-transplantation may be considered if FEV1 declines further.

The immunological mechanism underlying the development of both phenotypes is entirely different: NRAD includes neutrophils (BAL) and macrophages/lymphocytes (biopsy) leading to active fibrosis, while fBOS does not (no neutrophils in BAL and, until now, unclear histological characteristics). The present authors speculate that fBOS is initiated by a direct active fibrosis, of which the precise triggers remain unidentified. The central element in the differentiation of BOS may be explained by the severity and the duration of airway epithelial damage. However, NRAD could be triggered by repetitive, mild injury (even unrelated to rejection, such as infection/ colonisation, GER, air pollution and toxic inhalants [10, 11, 38]) of the epithelium that can be restored. Only persistent or nontreated damage will lead to fibrosis. In the development of fBOS, a single, very severe epithelial injury with a rather short duration may damage the epithelium to such an extent perhaps even leading to denudation, that repair and remodelling are out of balance. This may result in fibroproliferation without inflammation, which is supported by the study by JACKSON et al. [39], in which BOS is preceded by an acute event (e.g. AR or CMV infection) carrying a poor prognosis, with a high mortality due to OB. This central role of the epithelium and the severity of the injury in the onset of BOS have already been suggested by QU et al. [40] in a rat trachea allograft model.

However, this dichotomy does not fit into the current definition of BOS, which implies an irreversible loss of pulmonary function characterised by a neutrophilic inflammation leading to fibroproliferation of the airways. The NRAD phenotype questions this definition of BOS as it is, at least partially, reversible by azithromycin. Therefore, a reformulation or rephrasing of the definition seems essential. Perhaps NRAD has to be excluded from BOS and accepted as (nonspecific) activation of the innate immune system, resulting in inflammation that predisposes to BOS. In this way, the definition of BOS can be maintained as an irreversible loss of pulmonary function, characterised by a fibroproliferative disorder of the small airway ((fibrotic) active to inactive OB lesions) after exclusion of $A R$, infection, GER and responsiveness to azithromycin therapy. This implies that the neutrophilic inflammation should be evaluated as it is a prerequisite for azithromycin responsiveness; thus, BOS can then be characterised histologically as pure fibrotic active OB.
The current hypothesis has grown from the experience obtained in a single lung transplant centre. The present observations need to be evaluated and confirmed by the lung transplant sector. However, in a study by ABERNATHY et al. [41], this hypothesis can be seen when one reads between the lines. ABERNATHY et al. [41] carried out autopsies on seven patients following heart-lung transplantation and described three patients with acellular concentric fibrosis of the terminal bronchioles, which had started to develop $>6$ months after transplantation. The remaining four patients had cellular bronchiolitis obliterans on autopsy, which extended into the alveolar spaces with onset $<6$ months after transplantation. This was associated with innate inflammation as these patients had concurrent infections and aspiration. The first three patients may well represent fBOS, whereas the latter four resemble NRAD [41]. An important difference is that ABERNATHY et al. [41] describe a difference in histological localisation, as in this study NRAD developed distally in the airways (resembling bronchiolitis obliterans organising pneumonia), whereas fBOS was the "pure OB type". The present authors could not confirm this finding. DevOUASSOUx et al. [42] also described low BAL neutrophilia in patients who already had BOS for a longer period. This is in agreement with the current hypothesis that NRAD may evolve gradually and slowly from an early neutrophilic inflammation with inflammatory active lesions to "inactive" lesions. A recent study by LAMA et al. [43], in which emphasis was placed on time of onset (early or late) and slope of the FEV1 decline (rapid and gradual) in BOS patients, already alludes to the existence of different phenotypes of BOS. D'OVIDIO et al. [44] corroborated this finding of early and late BOS and also associated the early phenotype with neutrophilia/IL-8. Furthermore, D'OvIDIO et al. [44] found this inflammation to be related to GER, and more specifically to bile acid reflux.

This new dichotomy diverges from the accepted hypothesis of BOS being a homogenous entity, in which the response to azithromycin is related to the natural history of BOS (response at initial stage of BOS and nonresponse later on). However, the old classification is no longer acceptable since the present authors have phenotyped a form of BOS without neutrophils (present in the early days of BOS) and another form with neutrophils, which seem to predict the response to azithromycin [8]. In a series of patients with advanced BOS undergoing re-transplantation, MARTINU et al. [45] also demonstrated that $\mathrm{OB}$ was present in all explanted allografts but the degree of epithelial changes, fibrosis and inflammation varied considerably confirming heterogeneity and rejecting homogeneity among patients undergoing re-transplantation for BOS, potentially contributing to the variability of patient responses to treatment. The most important proof against the old hypothesis and in favour of the dichotomy is the consecutive appearance of NRAD and fBOS in some patients (patients 6 and 7 in the present study). If BOS had been a homogeneous entity, the second decline in FEV1 (fBOS) would not have appeared later on, as the neutrophilic inflammation was removed by azithromycin. Conversely, if it had been a new development of BOS, neutrophils should have been present at that time in the BAL, which was not the case. This appearance of fBOS after NRAD even raised speculation about NRAD being a risk factor for fBOS. Consequently, the idea of adding 
azithromycin to the maintenance therapy directly after transplantation to prevent NRAD and possibly the development of fBOS remains to be proven in a larger prospective study.

\section{Conclusion}

In conclusion, the present clinical observations, supported by research findings, have revealed a dichotomy in the clinical spectrum of bronchiolitis obliterans syndrome with neutrophilic reversible allograft dysfunction responding to azithromycin and fibroproliferative bronchiolitis obliterans syndrome not responding to azithromycin. This concept is reinforced by unique data obtained in bronchiolitis obliterans syndrome patients consisting of histological specimens, physical and radiological examinations, lung function testing and bronchoalveolar lavage examination. The acceptance of this dichotomy in bronchiolitis obliterans syndrome can improve current understanding of the heterogeneous pathological condition that constitutes bronchiolitis obliterans syndrome, thus encouraging a more accurate diagnosis and, ultimately, a better tailored treatment for each individual bronchiolitis obliterans syndrome patient. However, it is evident that this hypothesis needs further investigation and corroboration with different transplant centres around the world.

\section{REFERENCES}

1 Estenne M, Maurer JR, Boehler A, et al. Bronchiolitis obliterans syndrome 2001: an update of the diagnostic criteria. J Heart Lung Transplant 2002; 21: 297-310.

2 Gerhardt SG, McDyer JF, Girgis RE, Conte JV, Yang SC, Orens JB. Maintenance azithromycin therapy for bronchiolitis obliterans syndrome: results of a pilot study. Am J Respir Crit Care Med 2003; 168: 121-125.

3 Verleden GM, Dupont LJ. Azithromycin therapy for patients with bronchiolitis obliterans syndrome after lung transplantation. Transplantation 2004; 77: 1465-1467.

4 Yates B, Murphy DM, Forrest IA, et al. Azithromycin reverses airflow obstruction in established bronchiolitis obliterans syndrome. Am J Respir Crit Care Med 2005; 172: 772-775.

5 Shitrit D, Bendayan D, Gidon S, Saute M, Bakal I, Kramer MR. Long-term azithromycin use for treatment of bronchiolitis obliterans syndrome in lung transplant recipients. J Heart Lung Transplant 2005; 24: 1440-1443.

6 Crowley S, Egan JJ. Macrolide antibiotics and bronchiolitis obliterans following lung transplantation. Expert Rev Anti Infect Ther 2005; 3: 923-930.

7 Jaffe A, Bush A. Anti-inflammatory effects of macrolides in lung disease. Pediatr Pulmonol 2001; 31: 464-473.

8 Verleden GM, Vanaudenaerde BM, Dupont LJ, Van Raemdonck DE. Azithromycin reduces airway neutrophilia and IL-8 in patients with bronchiolitis obliterans syndrome. Am J Respir Crit Care Med 2006; 174: 566-570.

9 DiGiovine B, Lynch JP III, Martinez FJ, et al. Bronchoalveolar lavage neutrophilia is associated with obliterative bronchiolitis after lung transplantation: role of IL-8. J Immunol 1996; 157: 4194-4202.

10 Vanaudenaerde BM, Wuyts WA, Geudens N, et al. Macrolides inhibit IL17-induced IL8 and 8-isoprostane release from human airway smooth muscle cells. Am J Transplant 2007; 7: 76-81.
11 Zheng L, Whitford HM, Orsida B, et al. The dynamics and associations of airway neutrophilia post lung transplantation. Am J Transplant 2006; 6: 599-608.

12 Vos R, Vanaudenaerde BM, Dupont LJ, Van Raemdonck DE, Verleden GM. Transient airway colonization is associated with airway inflammation after lung transplantation. Am J Transplant 2007; 7: 1278-1287.

13 Nunley DR, Grgurich W, Iacono AT, et al. Allograft colonization and infections with pseudomonas in cystic fibrosis lung transplant recipients. Chest 1998; 113: 1235-1243.

14 Ward C, Camara M, Forrest I, et al. Preliminary findings of quorum signal molecules in clinically stable lung allograft recipients. Thorax 2003; 58: 444-446.

15 Tateda K, Comte R, Pechere JC, Kohler T, Yamaguchi K, Van Delden C. Azithromycin inhibits quorum sensing in Pseudomonas aeruginosa. Antimicrob Agents Chemother 2001; 45: 1930-1933.

16 von Rosensteil NA, Adam D. Macrolide antibacterials. Drug interactions of clinical significance. Drug Saf 1995; 13: 105-122.

17 Stanbrook MB, Kesten S. Bronchial hyperreactivity after lung transplantation predicts early bronchiolitis obliterans. Am J Respir Crit Care Med 1999; 160: 2034-2039.

18 Verleden GM, Dupont LJ, Van Raemdonck DE. Is it bronchiolitis obliterans syndrome or is it chronic rejection: a reappraisal? Eur Respir J 2005; 25: 221-224.

19 Palmer SM, Miralles AP, Howell DN, Brazer SR, Tapson VF, Davis RD. Gastroesophageal reflux as a reversible cause of allograft dysfunction after lung transplantation. Chest 2000; 118: 1214-1217.

20 Blondeau K, Mertens V, Vanaudenaerde BA, et al. Gastrooesophageal reflux and gastric aspiration in lung transplant patients with or without chronic rejection. Eur Respir J 2008; 31: 707-713.

21 Verleden GM, Besse T, Maes B. Successful conversion from cyclosporine to tacrolimus for gastric motor dysfunction in a lung transplant recipient. Transplantation 2002; 73: 1974-1976.

22 Verleden GM, Dupont LJ, Vanhaecke J, Daenen W, Van Raemdonck DE. Effect of azithromycin on bronchiectasis and pulmonary function in a heart-lung transplant patient with severe chronic allograft dysfunction: a case report. $J$ Heart Lung Transplant 2005; 24: 1155-1158.

23 Blondeau K, Mertens V, Vanaudenaerde BM, et al. Acid and non-acid gastroesophageal reflux and pepsin levels in BAL in lung transplant recipients with or without azithromycin treatment. J Heart Lung Transplant 2007; 26: S139-S140.

24 Murphy DM, Forrest IA, Ward C, et al. Effect of azithromycin on primary bronchial epithelial cells derived from stable lung allografts. Thorax 2007; 62: 834.

25 Kastelik JA, Peckham DG, Conway S. Azithromycin in cystic fibrosis. Eur Respir J 2005; 25: 771-772.

26 Boehler A, Kesten S, Weder W, Speich R. Bronchiolitis obliterans after lung transplantation: a review. Chest 1998; 114: 1411-1426.

27 Verbeken EK, Stewart S. Bronchiolitis obliterans syndrome, bronchiolitis obliterans in lung transplantation: will a pathologist and a physician ever meet? In: Verleden GM, Van Raemdonck D, Lerut T, Demedts M, eds. Surgery 
for Non-Neoplastic Disorders of the Chest: a Clinical Update. Eur Respir Mon 2004; 9: 55-65.

28 Sokoloff L. Pathology of rheumatoid arthritis and allied disorders. In: McCarty, DJ, ed. Arthritis and Allied Disorders. Philadelphia, Lea and Febiger, 1979; pp. 430448.

29 de Jong PA, Dodd JD, Coxson HO, et al. Bronchiolitis obliterans following lung transplantation: early detection using computed tomographic scanning. Thorax 2006; 61: 799-804.

30 Hoiby N. Diffuse panbronchiolitis and cystic fibrosis: East meets West. Thorax 1994; 49: 531-532.

31 Johnston SL, Blasi F, Black PN, Martin RJ, Farrell DJ, Nieman RB. The effect of telithromycin in acute exacerbations of asthma. N Engl J Med 2006; 354: 1589-1600.

32 Parnham MJ, Culic O, Erakovic V, et al. Modulation of neutrophil and inflammation markers in chronic obstructive pulmonary disease by short-term azithromycin treatment. Eur J Pharmacol 2005; 517: 132-143.

33 McCormack J, Bell S, Senini S, et al. Daily versus weekly azithromycin in cystic fibrosis patients. Eur Respir J 2007; 30: 487-495.

34 Saeki T, Ueda K, Tanigawara Y, Hori R, Komano T. Human P-glycoprotein transports cyclosporin A and FK506. J Biol Chem 1993; 268: 6077-6080.

35 Yee GC, McGuire TR. Pharmacokinetic drug interactions with cyclosporin (Part I). Clin Pharmacokinet 1990; 19: 319-332.

36 Mori T, Aisa Y, Nakazato T, Yamazaki R, Ikeda Y, Okamoto S. Tacrolimus-azithromycin interaction in a recipient of allogeneic bone marrow transplantation. Transpl Int 2005; 18: 757-758.

37 Gladue RP, Bright GM, Isaacson RE, Newborg MF. In vitro and in vivo uptake of azithromycin (CP-62,993) by phagocytic cells: possible mechanism of delivery and release at sites of infection. Antimicrob Agents Chemother 1989; 33: 277-282.

38 D'Ovidio F, Mura M, Ridsdale R, et al. The effect of reflux and bile acid aspiration on the lung allograft and its surfactant and innate immunity molecules SP-A and SP-D. Am J Transplant 2006; 6: 1930-1938.

39 Jackson CH, Sharples LD, McNeil K, Stewart S, Wallwork J. Acute and chronic onset of bronchiolitis obliterans syndrome (BOS): are they different entities? J Heart Lung Transplant 2002; 21: 658-666.

$40 \mathrm{Qu}$ N, de Vos P, Schelfhorst M, de Haan A, Timens W, Prop J. Integrity of airway epithelium is essential against obliterative airway disease in transplanted rat tracheas. $J$ Heart Lung Transplant 2005; 24: 882-890.

41 Abernathy EC, Hruban RH, Baumgartner WA, Reitz BA, Hutchins GM. The two forms of bronchiolitis obliterans in heart-lung transplant recipients. Hum Pathol 1991; 22: 1102-1110.

42 Devouassoux G, Drouet C, Pin I, et al. Alveolar neutrophilia is a predictor for the bronchiolitis obliterans syndrome, and increases with degree of severity. Transpl Immunol 2002; 10: 303-310.

43 Lama VN, Murray S, Lonigro RJ, et al. Course of FEV(1) after onset of bronchiolitis obliterans syndrome in lung transplant recipients. Am J Respir Crit Care Med 2007; 175: 1192-1198.

44 D'Ovidio F, Mura M, Tsang M, et al. Bile acid aspiration and the development of bronchiolitis obliterans after lung transplantation. J Thorac Cardiovasc Surg 2005; 129: 11441152.

45 Martinu T, Howell DN, Davis RD, Steele MP, Palmer SM. Pathologic correlates of bronchiolitis obliterans syndrome in pulmonary retransplant recipients. Chest 2006; 129: 1016-1023. 